PROCEEDINGS OF THE

AMERICAN MATHEMATICAL SOCIETY

Volume 139, Number 4, April 2011, Pages 1387-1396

S 0002-9939(2010)10573-7

Article electronically published on August 31, 2010

\title{
TOEPLITZ AND HANKEL OPERATORS ASSOCIATED WITH SUBDIAGONAL ALGEBRAS
}

\author{
BEBE PRUNARU
}

(Communicated by Mario Bonk)

\begin{abstract}
Let $\mathcal{M}$ be a $\sigma$-finite von Neumann algebra and let $\mathcal{A} \subset \mathcal{M}$ be a maximal subdiagonal algebra with respect to some faithful normal expectation $\mathcal{E}$ on $\mathcal{M}$. Let $\phi$ be a normal faithful $\mathcal{E}$-invariant state on $\mathcal{M}$, let $L^{2}(\mathcal{M}, \phi)$ be the non-commutative Lebesgue space in the sense of U. Haagerup, and consider the Hardy space $H^{2}(\mathcal{A}, \phi) \subset L^{2}(\mathcal{M}, \phi)$ associated with the pair $(\mathcal{A}, \phi)$. For each $x \in \mathcal{M}$, the Toeplitz operator $T_{x} \in B\left(H^{2}(\mathcal{A}, \phi)\right)$ and the Hankel operator $H_{x} \in B\left(H^{2}(\mathcal{A}, \phi), H^{2}(\mathcal{A}, \phi)^{\perp}\right)$ are defined as in the classical case of the unit circle. We show that the mapping $x \mapsto T_{x}$ is completely isometric on $\mathcal{M}$ and therefore $\sigma(x) \subset \sigma\left(T_{x}\right)$ for all $x \in \mathcal{M}$. We also show that $\left\|H_{x}\right\|=\operatorname{dist}(x, \mathcal{A})$ for every $x \in \mathcal{M}$.
\end{abstract}

\section{INTRODUCTION}

Subdiagonal algebras were introduced by W. Arveson in [1] with the purpose to provide a unifying approach to the study of several (apparently unrelated) classes of non-selfadjoint operator algebras, including algebras of matrix-valued analytic functions 12 and triangular algebras [17]. In the subsequent decades, a wealth of concrete classes of subdiagonal algebras, e.g. the non-selfadjoint (or analytic) crossed products 21] and other closely related algebras such as the analytic operator algebras [18, have been constructed and studied from various points of view, including factorization theory, invariant subspaces or maximality among $\sigma$-weakly closed subalgebras. Both [18] and 21], as well as [4, and the papers mentioned after Definition 1.3 contain a large number of relevant references on this subject.

Definition 1.1. Let $\mathcal{M}$ be a von Neumann algebra and let $\mathcal{E}: \mathcal{M} \rightarrow \mathcal{D}$ be a faithful normal conditional expectation onto a von Neumann subalgebra $\mathcal{D} \subset \mathcal{M}$. A $\sigma$-weakly closed subalgebra $\mathcal{A} \subset \mathcal{M}$ is said to be a subdiagonal algebra in $\mathcal{M}$ with respect to $\mathcal{E}$ if it satisfies the following conditions:

(i) the linear manifold $\left\{a+b^{*}: a, b \in \mathcal{A}\right\}$ is $\sigma$-weakly dense in $\mathcal{M}$;

(ii) $\mathcal{E}(a b)=\mathcal{E}(a) \mathcal{E}(b)$ for every $a, b \in \mathcal{A}$;

(iii) $\mathcal{A} \cap \mathcal{A}^{*}=\mathcal{D}\left(\right.$ where $\left.\mathcal{A}^{*}=\left\{a^{*}: a \in \mathcal{A}\right\}\right)$.

Received by the editors September 10, 2009 and, in revised form, April 26, 2010.

2010 Mathematics Subject Classification. Primary 46L51, 47B35; Secondary 47L25, 47L30.

Key words and phrases. Subdiagonal algebras, Toeplitz operators, Hankel operators, noncommutative Hardy spaces.

This research was partially supported by Grant PNII - Programme "Idei" (code 1194).

(C)2010 American Mathematical Society Reverts to public domain 28 years from publication 
The original definition from [1 does not assume that $\mathcal{A}$ is $\sigma$-weakly closed; however, as shown in [1, the $\sigma$-weak closure of a subdiagonal algebra as defined in that paper is subdiagonal as in the above definition.

If $\mathcal{A}$ is not properly contained in any other subdiagonal algebra of $\mathcal{M}$ with respect to the same conditional expectation $\mathcal{E}$, then $\mathcal{A}$ is called a maximal subdiagonal algebra in $\mathcal{M}$ (with respect to $\mathcal{E}$ ). It was proved in [1] that every subdiagonal algebra $\mathcal{A} \subset \mathcal{M}$ of a $\sigma$-finite von Neumann algebra is contained in exactly one maximal subdiagonal algebra $\mathcal{A}_{\max }$, which is defined by

$$
\mathcal{A}_{\text {max }}=\{x \in \mathcal{M}: \mathcal{E}(a x b)=\mathcal{E}(b x a)=0, \forall a \in \mathcal{A}, \forall b \in \mathcal{A} \cap \operatorname{Ker}(\mathcal{E})\} .
$$

Suppose now that there exists a faithful normal finite trace $\tau$ on $\mathcal{M}$ such that $\tau \circ \mathcal{E}=\tau$ on $\mathcal{M}$. In this case $\mathcal{A}$ is said to be a finite subdiagonal algebra in $\mathcal{M}$ (w.r.t. $\mathcal{E}$ ). It was proved in 7 that every finite subdiagonal algebra is maximal subdiagonal.

A fundamental result in the theory of subdiagonal algebras is the factorization theorem of $\mathrm{W}$. Arveson [1. It says that if $\mathcal{A} \subset \mathcal{M}$ is a finite maximal subdiagonal algebra of a finite von Neumann algebra, then every invertible element $x \in \mathcal{M}$ can be written as $x=u a$, where $u \in \mathcal{M}$ is unitary and both $a$ and $a^{-1}$ belong to $\mathcal{A}$.

In this paper we shall use the following terminology:

Definition 1.2. A quadruple $\{\mathcal{M}, \mathcal{A}, \mathcal{E}, \phi\}$ will be called subdiagonal if $\mathcal{M}$ is a $\sigma$ finite von Neumann algebra, $\mathcal{A} \subset \mathcal{M}$ is a maximal subdiagonal algebra with respect to a faithful normal conditional expectation $\mathcal{E}$ on $\mathcal{M}$ and $\phi$ is a faithful normal state on $\mathcal{M}$ such that $\phi \circ \mathcal{E}=\phi$. If, moreover, $\phi$ is tracial, then this quadruple will be called finite subdiagonal.

Let $\mathcal{M}$ be a $\sigma$-finite von Neumann algebra and let $\phi$ be a faithful normal state on $\mathcal{M}$. Let $L^{2}(\mathcal{M}, \phi)$ be the non-commutative $L^{2}$-space associated to $\mathcal{M}$ and $\phi$ in the sense of Haagerup [8, 9]. See also the recent survey [23] and [10. For our purposes in this paper it will be sufficient to think of $L^{2}(\mathcal{M}, \phi)$ as being the completion of $\mathcal{M}$ in the norm $\|x\|_{\phi}=\phi\left(x^{*} x\right)^{1 / 2}$ for $x \in \mathcal{M}$. Indeed, it is known that $L^{2}(\mathcal{M}, \phi)$ contains a distinguished vector $\xi_{0}$ which is both cyclic and separating for the algebra of all left multiplication operators $h \mapsto x h$ with elements from $\mathcal{M}$ and which also satisfies $\phi(x)=\left(x \xi_{0}, \xi_{0}\right)$ for every $x \in \mathcal{M}$.

Definition 1.3. Let $\{\mathcal{M}, \mathcal{A}, \mathcal{E}, \phi\}$ be a subdiagonal quadruple. The Hardy space $H^{2}(\mathcal{A}, \phi) \subset L^{2}(\mathcal{M}, \phi)$ is defined as the completion of $\mathcal{A}$ in the norm $\|\cdot\|_{\phi}$. Let $P$ be the orthogonal projection onto $H^{2}(\mathcal{A}, \phi)$.

(i) The Toeplitz map $\mathcal{T}_{(\mathcal{A}, \phi)}: \mathcal{M} \rightarrow B\left(H^{2}(\mathcal{A}, \phi)\right)$ is defined by $\mathcal{T}_{(\mathcal{A}, \phi)}(x) h=$ $P(x h), \quad h \in H^{2}(\mathcal{A}, \phi)$.

(ii) The Hankel map $\mathcal{H}_{(\mathcal{A}, \phi)}: \mathcal{M} / \mathcal{A} \rightarrow B\left(H^{2}(\mathcal{A}, \phi), H^{2}(\mathcal{A}, \phi)^{\perp}\right)$ is defined by $\mathcal{H}_{(\mathcal{A}, \phi)}(x+\mathcal{A}) h=(I-P)(x h), \quad h \in H^{2}(\mathcal{A}, \phi)$.

Hardy spaces associated to finite subdiagonal algebras have been studied intensively; see the recent survey [3] and the references therein. It turns out that most of the fundamental theorems in classical function theory associated to the Hardy spaces of a weak* Dirichlet algebra [26] have non-commutative versions in the context of finite subdiagonal algebras. Toeplitz and Hankel operators associated to finite subdiagonal algebras were studied in [19] and 20. Toeplitz and Hankel operators associated to non-selfadjoint crossed products have been studied in [24, 25] and [13. 
In this paper we show that both the Toeplitz and the Hankel maps associated to a subdiagonal algebra are isometric. For the case of a finite subdiagonal algebra $\mathcal{A} \subset \mathcal{M}$ the Hankel map is known to be isometric [24] and [19] (therefore extending a famous theorem of $\mathrm{Z}$. Nehari [22]), and the Toeplitz map is known to be isometric when restricted to $\mathcal{A}[19$. First we show that in the finite case the Toeplitz map is isometric on $\mathcal{M}$. We then employ a reduction scheme devised by $\mathrm{U}$. Haagerup together with a certain construction due to Q. Xu in order to extend these results to non-finite subdiagonal algebras. As a corollary we obtain an extension of the spectral inclusion theorem of P. Hartman and A. Wintner [11. In the finite subdiagonal case this extension was proved in [20].

\section{Preliminaries}

If $\mathcal{X}$ and $\mathcal{Y}$ are operator spaces and $\Psi: \mathcal{X} \rightarrow \mathcal{Y}$ is a linear mapping, then

$$
\Psi^{(n)}: M_{n}(\mathcal{X}) \rightarrow M_{n}(\mathcal{Y})
$$

will denote the map defined for each $n \geq 1$ by

$$
\Psi^{(n)}\left(\left[x_{i, j}\right]\right)=\left[\Psi\left(x_{i, j}\right)\right], \quad\left[x_{i, j}\right] \in M_{n}(\mathcal{X}) .
$$

The following easy lemma will be very useful in the sequel.

Lemma 2.1. Let $\{\mathcal{M}, \mathcal{A}, \mathcal{E}, \phi\}$ be a subdiagonal quadruple.

(i) The quadruple $\left\{M_{n}(\mathcal{M}), M_{n}(\mathcal{A}), \mathcal{E}^{(n)}, \phi \otimes t r_{n}\right\}$ is subdiagonal, where $t_{n}$ is the normalized trace on $M_{n}=M_{n}(\mathbb{C})$.

(ii) There exists a $*$-isomorphism

$$
\Theta: C^{*}\left\{\mathcal{T}_{(\mathcal{A}, \phi)}^{(n)}\left(M_{n}(\mathcal{M})\right)\right\} \rightarrow C^{*}\left\{\mathcal{T}_{\left(M_{n}(\mathcal{A}), \phi \otimes t r_{n}\right)}\left(M_{n}(\mathcal{M})\right)\right\}
$$

such that

$$
\Theta\left(\mathcal{T}_{(\mathcal{A}, \phi)}^{(n)}\left(\left[x_{i, j}\right]\right)\right)=\mathcal{T}_{\left(M_{n}(\mathcal{A}), \phi \otimes t r_{n}\right)}\left(\left[x_{i, j}\right]\right) \quad \forall\left[x_{i, j}\right] \in M_{n}(\mathcal{M}) .
$$

Proof. The proof of (i) is straightforward. As for (ii), we first see that the identity map on $M_{n}(\mathcal{A})$ extends to a unitary operator

$$
U_{n}: H^{2}\left(M_{n}(\mathcal{A}), \phi \otimes t r_{n}\right) \rightarrow H^{2}(\mathcal{A}, \phi) \otimes L^{2}\left(M_{n}, t r_{n}\right) .
$$

Next, consider the canonical embedding

$$
\rho_{n}: B\left(H^{2}(\mathcal{A}, \phi)\right) \otimes M_{n} \rightarrow B\left(H^{2}(\mathcal{A}, \phi) \otimes L^{2}\left(M_{n}, t r_{n}\right)\right) .
$$

It is now fairly easy to show that

$$
U_{n}^{*} \rho_{n}\left(\mathcal{T}_{(\mathcal{A}, \phi)}^{(n)}\left(\left[x_{i, j}\right]\right)\right) U_{n}=\mathcal{T}_{\left(M_{n}(\mathcal{A}), \phi \otimes t r_{n}\right)}\left(\left[x_{i, j}\right]\right)
$$

for every $\left[x_{i, j}\right] \in M_{n}(\mathcal{M})$. This completes the proof.

We shall use in the sequel the following result, which is a particular case of Theorem 4.4 from [2]

Theorem 2.2. Let $\{\mathcal{M}, \mathcal{A}, \mathcal{E}, \phi\}$ be a finite subdiagonal quadruple and let $\Psi: \mathcal{M} \rightarrow$ $\mathcal{B}$ be a unital and completely positive mapping into a unital $C^{*}$-algebra. Assume that its restriction to $\mathcal{A}$ is multiplicative and completely isometric. Then $\Psi$ itself is completely isometric.

We also need the following result from [19]. 
Theorem 2.3. Let $\{\mathcal{M}, \mathcal{A}, \mathcal{E}, \phi\}$ be a finite subdiagonal quadruple. Then the Toeplitz map $\mathcal{T}_{(\mathcal{A}, \phi)}$ is isometric when restricted to $\mathcal{A}$.

Corollary 2.4. If $\{\mathcal{M}, \mathcal{A}, \mathcal{E}, \phi\}$ is a finite subdiagonal quadruple, then the Toeplitz map $\mathcal{T}_{(\mathcal{A}, \phi)}$ is completely isometric on $\mathcal{M}$.

Proof. It follows from Lemma 2.1 that $\left\{M_{n}(\mathcal{M}), M_{n}(\mathcal{A}), \mathcal{E}^{(n)}, \phi \otimes t r_{n}\right\}$ is a finite subdiagonal quadruple for all $n \geq 1$. According to Theorem $2.3 \mathcal{T}_{\left(M_{n}(\mathcal{A}), \phi \otimes t r_{n}\right)}$ is isometric on $M_{n}(\mathcal{A})$. This, together with part (ii) of Lemma 2.1, shows that $\mathcal{T}_{(\mathcal{A}, \phi)}$ is completely isometric on $\mathcal{A}$. Since $\mathcal{T}_{(\mathcal{A}, \phi)}$ is obviously multiplicative on $\mathcal{A}$ it then follows from Theorem 2.2 that this map is completely isometric on $\mathcal{M}$.

The following extension of Nehari's theorem is essentially contained in 24 and stated in this form in 19 .

Theorem 2.5. Let $\{\mathcal{M}, \mathcal{A}, \mathcal{E}, \phi\}$ be a finite subdiagonal quadruple. Then the Hankel map $\mathcal{H}_{(\mathcal{A}, \phi)}$ is isometric on $\mathcal{M} / \mathcal{A}$.

\section{The HaAgerup Reduction method}

In the proof of our main result we shall use the Haagerup method of reduction to the finite case [8]. This method is frequently used in non-commutative integration theory; see [23] and [10]. For basic facts on crossed products and modular theory we refer to [16] and [27].

Theorem 3.1. Let $\mathcal{M} \subset B(H)$ be a $\sigma$-finite von Neumann algebra on some Hilbert space $H$ and let $\phi$ be a faithful normal state on $\mathcal{M}$. Let $\left\{\sigma_{t}^{\phi}\right\}$ be its modular automorphism group and let $G$ be the discrete subgroup of $\mathbb{R}$ defined by $G=\left\{m 2^{n}\right.$ : $n, m \in \mathbb{Z}\}$.

Consider the crossed product $\mathcal{R}=\mathcal{M} \rtimes_{\sigma^{\phi}} G$, where $\sigma^{\phi}$ is viewed as an automorphic representation of $G$, and let $\hat{\phi}$ be the dual weight of $\phi$, which in this case is a faithful normal state on $\mathcal{R}$ (because $G$ is discrete).

Then there exists an increasing sequence $\left\{\mathcal{R}_{n}\right\}_{n \geq 1}$ of von Neumann subalgebras of $\mathcal{R}$ with the following properties:

(a) Each $\mathcal{R}_{n}$ is finite.

(b) For each $n \geq 1$ there exists a faithful normal conditional expectation $\Phi_{n}$ : $\mathcal{R} \rightarrow \mathcal{R}$ onto $\mathcal{R}_{n}$ such that $\hat{\phi} \circ \Phi_{n}=\hat{\phi}$ (and hence $\sigma_{t}^{\hat{\phi}} \circ \Phi_{n}=\Phi_{n} \circ \sigma_{t}^{\hat{\phi}}$ ).

(c) $\bigcup_{n \geq 1} \mathcal{R}_{n}$ is $\sigma$-weakly dense in $\mathcal{R}$; more precisely, $\left\|\Phi_{n}(x)-x\right\|_{\hat{\phi}} \rightarrow 0$ for every $x \in \mathcal{R}$.

We need to recall how the sequence $\left\{\mathcal{R}_{n}\right\}$ is constructed. Let us first recall that if $\psi$ is a normal semifinite faithful weight on a von Neumann algebra $\mathcal{N}$, then its centralizer $\mathcal{N}_{\psi}$ is defined by

$$
\mathcal{N}_{\psi}=\left\{x \in \mathcal{N}: \sigma_{t}^{\psi}(x)=x,(\forall) t \in \mathbb{R}\right\} .
$$

If $\psi$ is finite we have that

$$
\mathcal{N}_{\psi}=\{x \in \mathcal{N}: \psi(x y)=\psi(y x),(\forall) y \in \mathcal{N}\} .
$$

The crossed product $\mathcal{R}=\mathcal{M} \rtimes_{\sigma^{\phi}} G$ is, by definition, the von Neumann algebra on the space $\ell^{2}(G, H)$ generated by the operators $\pi(x)$ and $\lambda(s)$ defined by

$$
(\pi(x) \xi)(t)=\sigma_{-t}^{\phi}(x) \xi(t), \quad x \in \mathcal{M}, \xi \in \ell^{2}(G, H), t \in G
$$


and

$$
(\lambda(s) \xi)(t)=\xi(t-s), \quad \xi \in \ell^{2}(G, H), t \in G, s \in G .
$$

These operators satisfy the commutation relation

$$
\pi\left(\sigma_{t}^{\phi}(x)\right)=\lambda(t) \pi(x) \lambda(t)^{*}, \quad x \in \mathcal{M}, t \in G .
$$

It follows that $\mathcal{R}$ is the $\sigma$-weak closure of the linear manifold

$$
\mathcal{R}_{0}=\left\{\sum_{j=1}^{n} \lambda\left(t_{j}\right) \pi\left(x_{j}\right), \quad t_{j} \in G, x_{j} \in \mathcal{M}\right\} .
$$

Recall that $\pi$ is a faithful normal representation of $\mathcal{M}$. Therefore we may and shall identify $\mathcal{M}$ with its image $\pi(\mathcal{M})$. We also recall that there exists a faithful normal conditional expectation $\Phi: \mathcal{R} \rightarrow \mathcal{M}$ which in this case for $t \in G$ and $x \in \mathcal{M}$ has the form $\Phi(\lambda(t) x)=x$ if $t=0$ and 0 elsewhere. The dual weight $\hat{\phi}=\phi \circ \Phi$ has the form $\hat{\phi}(\lambda(t) x)=\phi(x)$ if $t=0$ and 0 elsewhere. The modular automorphism group $\sigma_{t}^{\hat{\phi}}$ satisfies the following relation:

$$
\sigma_{t}^{\hat{\phi}}(x)=\lambda(t) x \lambda(t)^{*}, \quad x \in \mathcal{R}, t \in G .
$$

In particular, it follows that $\lambda(t) \in \mathcal{Z}\left(\mathcal{R}_{\hat{\phi}}\right)$ for every $t \in G$. It is then proved that for each $n \geq 1$ there exists a unique element $b_{n} \in \mathcal{Z}\left(\mathcal{R}_{\hat{\phi}}\right)$ with $0 \leq b_{n} \leq 2 \pi$ such that $\exp i b_{n}=\lambda\left(2^{-n}\right)$. By its construction, $b_{n}$ lies in the von Neumann subalgebra generated by $\{\lambda(t): t \in G\}$. One then defines $a_{n}=2^{n} b_{n}$ and

$$
\phi_{n}(x)=\hat{\phi}\left(e^{-a_{n}}\right)^{-1} \hat{\phi}\left(e^{-a_{n}} x\right), \quad x \in \mathcal{R} .
$$

We then have

$$
\sigma_{t}^{\phi_{n}}(x)=e^{-i t a_{n}} \sigma_{t}^{\hat{\phi}}(x) e^{i t a_{n}}, \quad x \in \mathcal{R}, t \in \mathbb{R},
$$

and one defines $\mathcal{R}_{n}=\mathcal{R}_{\phi_{n}}$. It then follows that $\phi_{n}$ is a faithful normal trace when restricted to $\mathcal{R}_{n}$. Finally the map $\Phi_{n}$ is defined by

$$
\Phi_{n}(x)=2^{n} \int_{0}^{2^{-n}} \sigma_{t}^{\phi_{n}}(x) d t, \quad x \in \mathcal{R} .
$$

We shall now describe a method that was devised in 28] in order to study subdiagonal algebras via Haagerup's reduction to the finite case. We shall keep the notation from Theorem 3.1] First recall the following result from [14].

Theorem 3.2. If $\{\mathcal{M}, \mathcal{A}, \mathcal{E}, \phi\}$ is a subdiagonal quadruple, then $\sigma_{t}^{\phi}(\mathcal{A}) \subset \mathcal{A}$ for all $t \in \mathbb{R}$.

Suppose now that $\mathcal{M}, \mathcal{A}, \mathcal{E}, \phi$ are given as in Theorem 3.2. Since $\phi$ is $\mathcal{E}$-invariant this implies that $\sigma_{t}^{\phi} \circ \mathcal{E}=\mathcal{E} \circ \sigma_{t}^{\phi}$. Let $\hat{\mathcal{A}} \subset \mathcal{R}$ be defined as the $\sigma$-weak closure of the linear manifold of all linear combinations of the form $\sum_{j=1}^{n} \lambda\left(t_{j}\right) x_{j}$ with $t_{j} \in G$ and $x_{j} \in \mathcal{A}$. Since, by Theorem 3.2, $\mathcal{A}$ is globally invariant under $\sigma_{t}^{\phi}$, it turns out that $\hat{\mathcal{A}}$ is a $\sigma$-weakly closed subalgebra of $\mathcal{R}$. Moreover, it can be shown that $\mathcal{E}$ can be extended to a faithful normal conditional expectation $\hat{\mathcal{E}}$ on $\mathcal{R}$ such that $\hat{\mathcal{E}}(\lambda(t) x)=\lambda(t) \mathcal{E}(x)$ for every $t \in G$ and $x \in \mathcal{M}$. It is proved in 28, that $\hat{\mathcal{A}}$ is a maximal subdiagonal algebra of $\mathcal{R}$ with respect to $\hat{\mathcal{E}}$. It is clear that $\hat{\phi} \circ \hat{\mathcal{E}}=\hat{\phi}$ and therefore $\hat{\mathcal{E}} \circ \sigma_{t}^{\hat{\phi}}=\sigma_{t}^{\hat{\phi}} \circ \hat{\mathcal{E}}$. For each $n \geq 1$, let $a_{n} \in \mathcal{R}$ be the element appearing in the construction of $\mathcal{R}_{n}$. Since $a_{n} \in \operatorname{Ran}(\hat{\mathcal{E}})$ it follows that $\phi_{n} \circ \hat{\mathcal{E}}=\phi_{n}$ and therefore $\hat{\mathcal{E}} \circ \sigma_{t}^{\phi_{n}}=\sigma_{t}^{\phi_{n}} \circ \hat{\mathcal{E}}$. In particular, it follows that $\Phi_{n} \circ \hat{\mathcal{E}}=\hat{\mathcal{E}} \circ \Phi_{n}$ for every $n \geq 1$. 
For each $n \geq 1$ let $\mathcal{A}_{n}=\mathcal{R}_{n} \cap \hat{\mathcal{A}}$. Then each $\mathcal{A}_{n}$ is a finite subdiagonal algebra of $\mathcal{R}_{n}$ with respect to the faithful normal conditional expectation $\mathcal{E}_{n}$ on $\mathcal{R}_{n}$ defined as the restriction of $\hat{\mathcal{E}}$ to $\mathcal{R}_{n}$. The union of all $\mathcal{A}_{n}$ 's is $\sigma$-weakly dense in $\hat{\mathcal{A}}$ and moreover $\mathcal{A}_{n}=\Phi_{n}(\hat{\mathcal{A}})$ for every $n \geq 1$. We refer to [28] for the proofs.

\section{THE MAIN RESUlT}

Let us now fix some notation. If $\mathcal{X}$ is a complex Banach space, then $\operatorname{Ball}(\mathcal{X})$ will denote the closed unit ball of $\mathcal{X}$. If $\mathcal{C} \subset \mathcal{X}$, then one denotes by $\overline{\operatorname{conv}}\{\mathcal{C}\}$ the closed convex hull of $\mathcal{C}$. For the remainder of this section we shall assume that $\{\mathcal{M}, \mathcal{A}, \mathcal{E}, \phi\}$ is a subdiagonal quadruple. Let

$$
\mathcal{A}_{\perp}=\left\{\psi \in \mathcal{M}_{*}: \psi(a)=0 \text { for every } a \in \mathcal{A}\right\},
$$

and let $\mathcal{A}_{0}=\{a \in \mathcal{A}: \mathcal{E}(a)=0\}$. Also let $\mathcal{A}_{0}^{*}=\left\{a^{*}: a \in \mathcal{A}_{0}\right\}$. For any $h, k \in$ $L^{2}(\mathcal{M}, \phi)$, we denote by $[h \otimes k]_{\phi}$ the element in $\mathcal{M}_{*}$ defined by

$$
\left\langle x,[h \otimes k]_{\phi}\right\rangle=(x h, k)_{\phi}, \quad x \in \mathcal{M},
$$

where $(\cdot, \cdot)_{\phi}$ is the scalar product in $L^{2}(\mathcal{M}, \phi)$. The following result is contained in the proof of Theorem 2.2 in [1].

Lemma 4.1. $\mathcal{A}_{0}^{*}$ is norm-dense in $H^{2}(\mathcal{A}, \phi)^{\perp}$.

The proof of the next lemma now becomes straightforward and it will be omitted.

Lemma 4.2. The following hold true:

(i) The Toeplitz map $\mathcal{T}_{(\mathcal{A}, \phi)}$ is isometric on $\mathcal{M}$ if and only if

$$
\overline{\operatorname{conv}}\left\{[a \otimes b]_{\phi}: a, b \in \mathcal{A} \text { and }\|a\|_{\phi},\|b\|_{\phi} \leq 1\right\}=\operatorname{Ball}\left(\mathcal{M}_{*}\right) .
$$

(ii) The Hankel map $\mathcal{H}_{(\mathcal{A}, \phi)}$ is isometric on $\mathcal{M} / \mathcal{A}$ if and only if

$$
\overline{\operatorname{conv}}\left\{[a \otimes b]_{\phi}: a \in \mathcal{A}, b \in \mathcal{A}_{0}^{*},\|a\|_{\phi},\|b\|_{\phi} \leq 1\right\}=\operatorname{Ball}\left(\mathcal{A}_{\perp}\right) .
$$

The main result of this paper is the following:

Theorem 4.3. Let $\{\mathcal{M}, \mathcal{A}, \mathcal{E}, \phi\}$ be a subdiagonal quadruple.

(i) The Toeplitz map $\mathcal{T}_{(\mathcal{A}, \phi)}$ is isometric on $\mathcal{M}$.

(ii) The Hankel map $\mathcal{H}_{(\mathcal{A}, \phi)}$ is isometric on $\mathcal{M} / \mathcal{A}$.

Proof. (i) The case of finite subdiagonal algebras is contained in Corollary 2.4 We now go to the general case. In what follows we shall freely use the notation and constructions from the previous sections. First of all, let us point out that $a_{n} \in \mathcal{A}_{n}$ for every $n \geq 1$ as one can easily see from the construction of $\mathcal{A}_{n}$. Let us denote by $\tau_{n}$ the restriction of $\phi_{n}$ to $\mathcal{R}_{n}$ and by $\psi_{n}$ the restriction of $\hat{\phi}$ to $\mathcal{R}_{n}$. It is clear that both $\psi_{n}$ and $\tau_{n}$ are $\mathcal{E}_{n}$-invariant faithful normal states on $\mathcal{R}_{n}$. For each $n \geq 1$ we know from the finite case that the Toeplitz map $\mathcal{T}_{\left(\mathcal{A}_{n}, \tau_{n}\right)}$ is isometric on $\mathcal{R}_{n}$. Recall that

$$
\phi_{n}(x)=\hat{\phi}\left(e^{-a_{n}}\right)^{-1} \hat{\phi}\left(e^{-a_{n}} x\right) \quad \forall x \in \mathcal{R} .
$$

Since $a_{n} \in \mathcal{R}_{\hat{\phi}}$ it follows that for every $x \in \mathcal{R}_{n}$ we have

$$
\tau_{n}(x)=\hat{\phi}\left(e^{-a_{n}}\right)^{-1} \psi_{n}\left(e^{-a_{n} / 2} x e^{-a_{n} / 2}\right) .
$$

This shows that the mapping

$$
\mathcal{R}_{n} \ni x \mapsto \hat{\phi}\left(e^{-a_{n}}\right)^{-1 / 2} x e^{-a_{n} / 2} \in \mathcal{R}_{n}
$$


extends to a unitary operator

$$
\Gamma_{n}: L^{2}\left(\mathcal{R}_{n}, \tau_{n}\right) \rightarrow L^{2}\left(\mathcal{R}_{n}, \psi_{n}\right)
$$

which intertwines the corresponding GNS representations of $\mathcal{R}_{n}$. Moreover, since $a_{n} \in \mathcal{A}_{n}$ we see that

$$
\Gamma_{n}\left(H^{2}\left(\mathcal{A}_{n}, \tau_{n}\right)\right)=H^{2}\left(\mathcal{A}_{n}, \psi_{n}\right) .
$$

This shows that for each $x \in \mathcal{R}_{n}$ the operators $\mathcal{T}_{\left(\mathcal{A}_{n}, \tau_{n}\right)}(x)$ and $\mathcal{T}_{\left(\mathcal{A}_{n}, \psi_{n}\right)}(x)$ are unitarily equivalent. It then follows that

$$
\overline{\operatorname{conv}}\left\{[a \otimes b]_{\psi_{n}}: a, b \in \mathcal{A}_{n},\|a\|_{\psi_{n}},\|b\|_{\psi_{n}} \leq 1\right\}=\operatorname{Ball}\left(\mathcal{R}_{n *}\right)
$$

Let $\pi_{n}: \mathcal{R}_{n *} \rightarrow \mathcal{R}_{*}$ be the predual map of $\Phi_{n}$, that is, $\pi_{n}^{*}=\Phi_{n}$. Then $\pi_{n}$ is clearly isometric and moreover

$$
\pi_{n}\left([a \otimes b]_{\psi_{n}}\right)=[a \otimes b]_{\hat{\phi}}, \quad a, b \in \mathcal{R}_{n} .
$$

In addition, since $\Phi_{n}(x) \rightarrow x \sigma$-weakly, it follows that $\bigcup_{n \geq 1} \pi_{n}\left(\mathcal{R}_{n *}\right)$ is norm dense in $\mathcal{R}_{*}$. This shows that

$$
\overline{\operatorname{conv}}\left\{[a \otimes b]_{\hat{\phi}}: a, b \in \hat{\mathcal{A}} \text { and }\|a\|_{\hat{\phi}},\|b\|_{\hat{\phi}} \leq 1\right\}=\operatorname{Ball}\left(\mathcal{R}_{*}\right) .
$$

Let $u=\sum_{j=1}^{n} \lambda\left(t_{j}\right) a_{j}$ and $v=\sum_{j=1}^{n} \lambda\left(t_{j}\right) b_{j}$ with $t_{j} \in G$ distinct points and $a_{j}, b_{j} \in \mathcal{A}$. Using the properties of $\Phi$ one can easily show that if $\pi_{*}: \mathcal{R}_{*} \rightarrow \mathcal{M}_{*}$ denotes the predual map of the canonical injection of $\mathcal{M}$ in $\mathcal{R}$, then

$$
\pi_{*}\left([u \otimes v]_{\hat{\phi}}\right)=\sum_{j=1}^{n}\left[a_{j} \otimes b_{j}\right]_{\phi} .
$$

Moreover, $\|u\|_{\hat{\phi}}^{2}=\sum_{j=1}^{n}\left\|a_{j}\right\|_{\phi}^{2}$ and similarly for $v$. In addition,

$$
\pi_{*}\left(\operatorname{Ball}\left(\mathcal{R}_{*}\right)\right)=\operatorname{Ball}\left(\mathcal{M}_{*}\right) .
$$

Since the set of all linear combinations of the form $\sum_{j=1}^{n} \lambda\left(t_{j}\right) a_{j}$ with $t_{j} \in G$ distinct points and $a_{j} \in \mathcal{A}$ for $1 \leq j \leq n$ is norm dense in $H^{2}(\hat{\mathcal{A}}, \hat{\phi})$, it follows that

$$
\overline{\operatorname{conv}}\left\{[a \otimes b]_{\phi}: a, b \in \mathcal{A} \text { and }\|a\|_{\phi},\|b\|_{\phi} \leq 1\right\}=\operatorname{Ball}\left(\mathcal{M}_{*}\right) .
$$

This shows that the Toeplitz map $\mathcal{T}_{(\mathcal{A}, \phi)}$ is isometric on $\mathcal{M}$.

(ii) The finite case is contained in Theorem 2.5. Let $n \geq 1$. The properties of the unitary operator $\Gamma_{n}$ constructed above show that the operators $\mathcal{H}_{\left(\mathcal{A}_{n}, \tau_{n}\right)}\left(x+\mathcal{A}_{n}\right)$ and $\mathcal{H}_{\left(\mathcal{A}_{n}, \psi_{n}\right)}\left(x+\mathcal{A}_{n}\right)$ are unitarily equivalent for every $x \in \mathcal{R}_{n}$. Since by the finite case the Hankel map $\mathcal{H}_{\left(\mathcal{A}_{n}, \tau_{n}\right)}$ is isometric on $\mathcal{R}_{n} / \mathcal{A}_{n}$, it follows that

$$
\overline{\operatorname{conv}}\left\{[a \otimes b]_{\psi_{n}}, a \in \mathcal{A}_{n}, b \in\left(\mathcal{A}_{n}\right)_{0}^{*},\|a\|_{\psi_{n}},\|b\|_{\psi_{n}} \leq 1\right\}=\operatorname{Ball}\left(\left(\mathcal{A}_{n}\right)_{\perp}\right) .
$$

Now, let us recall that $\pi_{n}: \mathcal{R}_{n *} \rightarrow \mathcal{R}_{*}$ denotes the predual map of $\Phi_{n}$. It turns out that $\pi_{n}\left(\left(\mathcal{A}_{n}\right)_{\perp}\right) \subset \hat{\mathcal{A}}_{\perp}$ and the union $\bigcup_{n>1} \pi_{n}\left(\left(\mathcal{A}_{n}\right)_{\perp}\right)$ is norm dense in $\hat{\mathcal{A}}_{\perp}$ because $\mathcal{A}_{n}=\Phi_{n}(\hat{\mathcal{A}})$ and $\Phi_{n}(x) \rightarrow x \sigma$-weakly for every $x \in \mathcal{R}$. Moreover it is obvious that $\left(\mathcal{A}_{n}\right)_{0} \subset \hat{\mathcal{A}}_{0}$; therefore

$$
\overline{\operatorname{conv}}\left\{[a \otimes b]_{\hat{\phi}}, a \in \hat{\mathcal{A}}, b \in\left(\hat{\mathcal{A}}_{0}\right)^{*},\|a\|_{\hat{\phi}},\|b\|_{\hat{\phi}} \leq 1\right\}=\operatorname{Ball}\left(\hat{\mathcal{A}}_{\perp}\right) .
$$

Since $\mathcal{E}$ and $\hat{\mathcal{E}}$ are idempotent maps, we see that $\mathcal{A}_{0}=(I-\mathcal{E})(\mathcal{A})$ and similarly for $\hat{\mathcal{A}}_{0}$. It then follows that the set of all linear combinations of the form $\sum_{j=1}^{n} \lambda\left(t_{j}\right) a_{j}$ with $a_{j} \in \mathcal{A}_{0}$ and $t_{j} \in G$ is $\sigma$-weakly dense in $\hat{\mathcal{A}}_{0}$. Moreover

$$
\pi_{*}\left(\operatorname{Ball}\left(\hat{\mathcal{A}}_{\perp}\right)\right)=\operatorname{Ball}\left(\mathcal{A}_{\perp}\right) .
$$


It then follows that

$$
\overline{\operatorname{conv}}\left\{[a \otimes b]_{\phi}: a \in \mathcal{A}, b \in \mathcal{A}_{0}^{*},\|a\|_{\phi},\|b\|_{\phi} \leq 1\right\}=\operatorname{Ball}\left(\mathcal{A}_{\perp}\right)
$$

Therefore $\mathcal{H}_{(\mathcal{A}, \phi)}$ is isometric on $\mathcal{M} / \mathcal{A}$.

We close with a corollary providing versions of some classical results on the invertibility of Toeplitz operators; see [6].

Corollary 4.4. Suppose that $\{\mathcal{M}, \mathcal{A}, \mathcal{E}, \phi\}$ are given as in Theorem 4.3. Denote $T_{x}=\mathcal{T}_{(\mathcal{A}, \phi)}(x)$ and $H_{x}=\mathcal{H}_{(\mathcal{A}, \phi)}(x+\mathcal{A})$ for $x \in \mathcal{M}$. Let $g l(\mathcal{A})$ be the group of invertible elements of $\mathcal{A}$.

(i) There exists a (unique) *-homomorphism

$$
\Pi: C^{*}\left\{\mathcal{T}_{(\mathcal{A}, \phi)}(\mathcal{M})\right\} \rightarrow \mathcal{M}
$$

such that $\Pi\left(T_{x}\right)=x$ for every $x \in \mathcal{M}$. In particular, $\sigma(x) \subset \sigma\left(T_{x}\right)$ for every $x \in \mathcal{M}$.

(ii) Let $a \in \mathcal{A}$. Then $T_{a}$ is invertible if and only if $a \in g l(\mathcal{A})$.

(iii) Let $v \in \mathcal{M}$ be an isometry. Then $T_{v}$ is left invertible if and only if $\operatorname{dist}(v, \mathcal{A})<1$.

(iv) Let $u \in \mathcal{M}$ be unitary. Then $T_{u}$ is invertible if and only if $\operatorname{dist}(u, g l(\mathcal{A}))<$ 1.

(v) If $x \in \mathcal{M}$ is invertible and if $x$ can be written as $x=u a$ with $u \in \mathcal{M}$ unitary and $a \in \operatorname{gl}(\mathcal{A})$, then $T_{x}$ is invertible iff $\operatorname{dist}(u, g l(\mathcal{A}))<1$.

Proof. (i). If we apply Theorem 4.3 to $\left\{M_{n}(\mathcal{M}), M_{n}(\mathcal{A}), \mathcal{E}^{(n)}, \phi \otimes t r_{n}\right\}$ (see the preliminary section for the notation), we infer that the associated Toeplitz map is isometric on $M_{n}(\mathcal{M})$ for every $n \geq 1$. Now we may apply Lemma 2.1 and conclude that the Toeplitz map on $H^{2}(\mathcal{A}, \phi)$ is completely isometric on $\mathcal{M}$. Now the existence of $\Pi$ easily follows from Theorem 4.1 in 5 .

(ii). If $a \in g l(\mathcal{A})$, then $T_{a}$ is invertible because the Toeplitz map is multiplicative on $\mathcal{A}$. Suppose now that $T_{a}$ is invertible. It then follows from (i) that $a$ is invertible in $\mathcal{M}$. Let $b \in \mathcal{M}$ be its inverse. Let $g \in H^{2}(\mathcal{A}, \phi)$ and let $f \in H^{2}(\mathcal{A}, \phi)$ such that $T_{a} f=g$. It then follows that $a f=g$; therefore $f=b g$. Now since $\mathcal{A}$ is maximal subdiagonal it follows from part (ii) of Theorem 4.3 (see also Theorem 2.2 from [15]) that

$$
\mathcal{A}=\left\{x \in \mathcal{M}: x\left(H^{2}(\mathcal{A}, \phi)\right) \subset H^{2}(\mathcal{A}, \phi)\right\} .
$$

This implies that $b \in \mathcal{A}$.

(iii). Let $f \in H^{2}(\mathcal{A}, \phi)$. Then

$$
\left\|T_{v} f\right\|^{2}=\|f\|^{2}-\left\|H_{v} f\right\|^{2} .
$$

This together with Theorem 4.3 shows that $T_{v}$ is bounded below iff $\operatorname{dist}(v, \mathcal{A})<1$.

(iv). Suppose now that $u \in \mathcal{M}$ is unitary. Assume that $T_{u}$ is invertible and let $a \in \mathcal{A}$ such that $\|u-a\|<1$. Then $\left\|1-u^{*} a\right\|<1$; therefore $\left\|I-T_{u}^{*} T_{a}\right\|<1$. This shows that $T_{a}$ is invertible; therefore $a \in \operatorname{gl}(\mathcal{A})$.

Conversely let us suppose that $\|u-a\|<1$ for some $a \in g l(\mathcal{A})$. Then $T_{a}$ is invertible and $\left\|I-T_{u}^{*} T_{a}\right\|<1$, which shows that $T_{u}$ is invertible.

(v). This follows easily from the previous items. 


\section{REFERENCES}

[1] W. B. Arveson, Analyticity in operator algebras, Amer. J. Math. 89 (1967), 578-642. MR0223899 (36:6946)

[2] D. P. Blecher and L. E. Labuschagne, Logmodularity and isometries of operator algebras, Trans. Amer. Math. Soc. 355 (2003), 1621-1646. MR.1946408 (2004c:46113)

[3] D. P. Blecher and L. E. Labuschagne, Von Neumann algebraic $H^{p}$ theory, Function spaces, 89-114, Contemp. Math., 435, Amer. Math. Soc., Providence, RI, 2007. MR2359421 (2008h:46027)

[4] D. P. Blecher and C. Le Merdy, Operator algebras and their modules-an operator space approach, Oxford Univ. Press, Oxford, 2004. MR.2111973 (2006a:46070)

[5] M. D. Choi and E. G. Effros, The completely positive lifting problem for $C^{*}$-algebras, Ann. of Math. (2) 104 (1976), 585-609. MR0417795 (54:5843)

[6] R.G. Douglas, Banach Algebra Techniques in Operator Theory, second edition. Graduate Texts in Mathematics, 179. Springer-Verlag, New York, 1998. MR.1634900 (99c:47001)

[7] R. Exel, Maximal subdiagonal algebras, Amer. J. Math. 110 (1988), 775-782. MR955297 (90b:46114)

[8] U. Haagerup, Noncommutative integration theory, unpublished manuscript, 1978.

[9] U. Haagerup, $L^{p}$-spaces associated with an arbitrary von Neumann algebra. In Algèbres d'opérateurs et leurs applications en physique mathématique (Proc. Colloq., Marseille, 1977), volume 274 of Colloq. Internat. CNRS, pages 175-184. CNRS, Paris, 1979. MR560633 (81e:46050)

[10] U. Haagerup, M. Junge and Q. Xu, A reduction method for noncommutative Lp-spaces and applications, Trans. Amer. Math. Soc. 362 (2010), 2125-2165. MR2574890

[11] P. Hartman and A. Wintner, On the spectra of Toeplitz's matrices. Amer. J. Math. 72 (1950), 359-366. MR0036936 (12:187a)

[12] H. Helson and D. Lowdenslager, Prediction theory and Fourier series in several variables. I, Acta Math. 99 (1958), 165-202. MR0097688 (20:4155)

[13] Y. Imina and K.-S. Saito, Hankel operators associated with analytic crossed products, Canad. Math. Bull. 37 (1994), 75-81. MR.1261560 (94k:47024)

[14] G.-X. Ji, T. Ohwada and K.-S. Saito, Certain structure of subdiagonal algebras, J. Operator Theory 39 (1998), 309-317. MR.1620570 (99b:46093)

[15] G.-X. Ji and K.-S. Saito, Factorization in subdiagonal algebras, J. Funct. Anal. 159 (1998), 191-202. MR1654186 (99k:46106)

[16] R.V. Kadison and J.R. Ringrose, Fundamentals of the Theory of Operator Algebras. II, Academic Press, 1986. MR859186 (88d:46106)

[17] R.V. Kadison and I.M. Singer, Triangular operator algebras. Fundamentals and hyperreducible theory. Amer. J. Math. 82 (1960), 227-259. MR0121675 (22:12409)

[18] R. Loebl and P. Muhly, Analyticity and flows in von Neumann algebras, J. Funct. Anal. 29 (1978), 214-252. MR504460 (81h:46080)

[19] M. Marsalli, Noncommutative $H^{2}$ spaces, Proc. Amer. Math. Soc. 125 (1997), 779-784. MR.1350954 (97e:46089)

[20] M. Marsalli and G. West, Toeplitz operators with noncommuting symbols, Integr. Equ. Oper. Theory 32 (1998), 65-74. MR1643198 (2000a:47058)

[21] M. McAsey, P. Muhly, and K.-S. Saito, Nonselfadjoint crossed products (invariant subspaces and maximality), Trans. Amer. Math. Soc. 248 (1979), 381-410. MR.522266 (80j:46101b)

[22] Z. Nehari, On bounded bilinear forms. Ann. of Math. (2) 65 (1957), 153-162. MR0082945 (18:633f)

[23] G. Pisier and Q. Xu. Non-commutative $L^{p}$-spaces. In Handbook of the Geometry of Banach Spaces, Vol. 2, 1459-1517. North-Holland, Amsterdam, 2003. MR,1999201 (2004i:46095)

[24] K.-S. Saito, Toeplitz operators associated with analytic crossed products, Math. Proc. Cambridge Philos. Soc. 108 (1990), 539-549. MR1068455 (91m:46109)

[25] K.-S. Saito, Toeplitz operators associated with analytic crossed products. II: (invariant subspaces and factorisation), Integral Equations Operator Theory 14 (1991), 251-275. MR1090704 (92e:46134)

[26] T. P. Srinivasan and J. Wang, Weak *-Dirichlet algebras. 1966 Function Algebras (Proc. Internat. Sympos. on Function Algebras, Tulane Univ., 1965), pp. 216-249, Scott-Foresman, Chicago, Ill. MR0198282(33:6441) 
[27] M. Takesaki, Theory of operator algebras, II. Springer-Verlag, Berlin, 2003. MR1943006 (2004g:46079)

[28] Q. Xu, On the maximality of subdiagonal algebras, J. Operator Theory 54 (2005), 137-146. MR2168864 (2006h:46058)

Institute of Mathematics "Simion Stollow" of the Romanian Academy, P.O. Box 1-764, RO-014700 Bucharest, Romania

E-mail address: Bebe.Prunaru@imar.ro 